\title{
Archipel
}

ARCHIPEL Études interdisciplinaires sur le monde insulindien

$97 \mid 2019$

Varia

\section{Sejarah Gerakan Kiri Indonesia untuk Pemula}

\section{Gloria Truly Estrelita}

\section{(2) OpenEdition}

\section{Journals}

Electronic version

URL: https://journals.openedition.org/archipel/1172

DOI: 10.4000/archipel. 1172

ISSN: 2104-3655

\section{Publisher}

Association Archipel

Printed version

Date of publication: 11 June 2019

Number of pages: $317-320$

ISBN: 978-2-910513-81-8

ISSN: 0044-8613

\section{Electronic reference}

Gloria Truly Estrelita, "Sejarah Gerakan Kiri Indonesia untuk Pemula", Archipel [Online], 97 | 2019, Online since, connection on 15 September 2021. URL: http://journals.openedition.org/archipel/1172 ; DOI: https://doi.org/10.4000/archipel.1172 
Durant son exil à Buru, certains événements l'ont amené à se remémorer son enfance, ses parents et sa vie avant la détention. Il y fait allusion, et cela nous permet d'imaginer tout ce qu'il a perdu, bien qu'il s'exprime de manière légère, sans regrets. Il évoque, par exemple, la maison dont il devait hériter, aujourd'hui habitée par un militaire de haut rang', et sa fiancée qui n'a plus donné de nouvelles depuis 1969 (p. 354).

Le rapport de la Commission Nationale des Droits de l'Homme (Komnas HAM) a enregistré que 11500 prisonniers ont été envoyés à Buru. Ces prisonniers ont fourni un travail forcé sans salaire, dans des entrepôts, au bureau du commandement militaire, dans une usine de ciment, à la construction d'hébergements dans le camp et de barrages sur l'île, et à l'implantation de rizières. Selon l'historien Asvi Warman Adam, il y avait 23 camps de concentration à Buru, chaque camp hébergeant 500 détenus. Quand les prisonniers politiques sont arrivés, l'île de Buru était encore une jungle. À partir de 1974, les prisonniers ont travaillé dur à la construction de barrages et de milliers d'hectares de rizière $^{10}$. Dans les années 90 , l'île de Buru est devenue une région de production de riz importante pour l'Indonésie. Malgré toutes les contributions des prisonniers politiques à Buru, le gouvernement ne reconnaît toujours pas la souffrance qu'ils ont endurée. Dans le but du combat contre l'oubli, la publication des mémoires des prisonniers politiques et des autres victimes est importante. Elle contribue à donner des sources d'information aux historiens pour leurs études et aux Indonésiens en général pour prendre conscience de leur histoire, afin d'éviter de commettre les mêmes erreurs.

Mulyandari Coetmeur

Sejarah Gerakan Kiri Indonesia untuk Pemula (« Histoire des mouvements de gauche indonésiens pour débutants », oleh Yayak Yatmaka dan 31 penulis lain, Bandung: Ultimus, 2016, 21 x 29,5 cm, cxxxiv-528 p., ISBN : 978-602-8331-65-4.

Durant plus de trente ans, l'écriture de l'histoire indonésienne a été contrôlée par le gouvernement de Soeharto. Les faits et la perspective historique ont été construits en fonction des intérêts du groupe au pouvoir à cette époque. Cet ouvrage, rédigé par un collectif, est un livre d'histoire illustré dont le but est de présenter des faits et une perspective historique nouveaux sur un groupe qui a jusqu'à présent été éliminé par l'État : la gauche. Le livre se divise en quatre chapitres correspondant aux phases de l'histoire nationale.

Le premier chapitre retrace l'apparition du colonialisme et la naissance du mouvement révolutionnaire. L'arrivée des colons européens a été suivie par la confiscation des droits de propriété et a entraîné la transformation d'une firme commerciale, la compagnie néerlandaise des Indes orientales (VOC), en organisme politique. Le lecteur est invité à voir en images le développement du capitalisme dans l'État colonial, le système des plantations obligatoires, le travail forcé, l'émergence d'une classe ouvrière, la résistance à l'impérialisme et la création du Indische Sociaal Democratische Vereeniging ou ISDV (1'Association social-démocrate des Indes), qui devint par la suite le Parti communiste indonésien (PKI).

9. Après 1966, des propriétés privées appartenant à des détenus furent réquisitionnés par des militaires, les droits de propriété étant transférés de manière illégale.

10. Rapport International People Tribunal on Human Rights Violation in 1965-1966. 


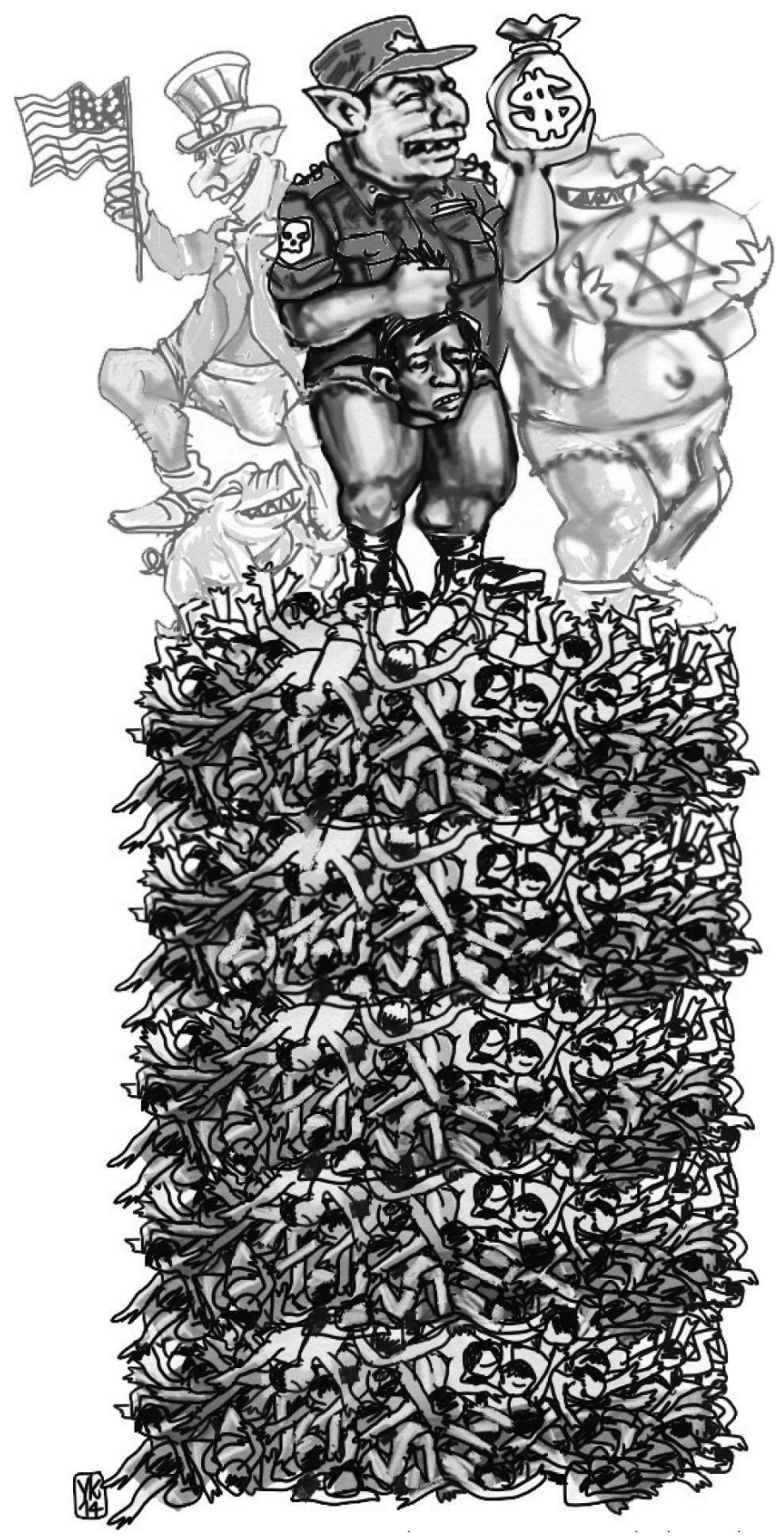

Sejarah Gerakan Kiri Indonesia untuk Pemula, p. 367.

(Avec l'aimable autorisation des éditions Ultimus, Bandung.) 
Le deuxième chapitre traite de la dynamique de la gauche, entre 1920 et 1965 , notamment les mouvements populaires, comme les révoltes paysannes, les grèves de cheminots et la création d'organisations anti-coloniales. Puis le PKI, le premier parti à utiliser le nom "Indonésie " au lieu de "Indes ", conduisit la première rébellion nationale contre le colonialisme néerlandais, à Java en 1926 et à Sumatra en 1927. Les illustrations montrent, une par une, les formes de sanctions infligées à ceux qui étaient accusés de rébellion contre le gouvernement colonial, dont la cruauté provoqua justement une résistance accrue du PKI envers ce qu'il appelait la « terreur blanche ». Ce chapitre rapporte également les agissements de la gauche sur la scène politique, les relations du PKI avec les groupes musulmans, Soekarno en tant que chef d'État, ainsi que les tentatives de pays étrangers d'intervenir dans la politique indonésienne.

Le troisième chapitre est consacré à la tragédie de 1965 et à l'écrasement de la gauche indonésienne par les militaires, assistés par des pays du Bloc occidental après les événements : arrestations massives, tortures, incarcérations, travail forcé, exil et massacres de toutes les personnes considérées comme impliquées dans des mouvements de gauche. La stigmatisation des prisonniers politiques comme traîtres à la nation, après leur libération, entraîna une limitation de leurs droits dans les domaines économique, social et politique. Les illustrations montrent les diverses formes de violences et aident à comprendre le modèle de conspiration politique de la guerre froide qui a conduit l'Indonésie dans une phase historique sanglante. Le livre tente de montrer l'importance de cette tragédie d'un point de vue historique, en ceci qu'elle a transformé le paysage social, culturel, politique et économique du pays.

Le quatrième chapitre expose la continuation des actes de violence après 1965: disparition de militants, interdiction de livres, contrôle de la presse, interdiction de débats et de toutes activités liées à l'histoire de la gauche. Les illustrations rappellent l'héritage mémoriel légué par le régime de Soeharto, notamment le discours anticommuniste et la propagande sur le fantôme du communisme, autant de choses qui survivent jusqu'à aujourd'hui.

Ce livre, fourmillant d'illustrations et d'une typographie caractéristique des bandes dessinées, paraît facile à lire, mais le texte n'est pas aussi simple que le donne à croire la couverture, faite d'une mosaïque d'images multicolores. Les dessins de type caricatural comprennent de nombreuses images violentes : corps nus et ensanglantés, tortures, viols, ou encore exécutions sadiques des individus " de gauche », ainsi que des illustrations symboliques : militaire debout sur un amoncellement de cadavres, ou gardien de l'ordre au faciès cruel et avec des oreilles sataniques dénotant son inhumanité (voir l'illustration).

Le lecteur de cette « Histoire de la gauche » est convié à considérer une narration de l'histoire différente de celle diffusée jusqu'à présent. Par exemple, nous apprenons l'utilisation du code « Jakarta, Jakarta » par le clan de Pinochet lors de son coup d'État contre le gouvernement de gauche d'Allende, au Chili, en 1973. Le livre donne également un point de vue nouveau sur l'Affaire de Madiun, en 1948, à savoir qu'elle fut pour les auteurs suscitée par la politique de "Réorganisation et Rationalisation » (Re-Ra) appliquée par le Cabinet Hatta en vue de réduire les effectifs de l'armée. Le mouvement de résistance du PKI à cette politique aboutit à des violences : enlèvement de militants du PKI, assassinats, terreur, qui causèrent un conflit ouvert entre le PKI et le gouvernement. La cause du conflit, telle que décrite ici, n'est pas celle d'une tentative de coup d'état du PKI présentée par l'histoire officielle. 
On regrette cependant que le livre n'expose pas de façon critique les événements jalonnant l'histoire de la gauche, le fait par exemple que la politique Re-Ra avait tout d'abord été supportée par le premier ministre, Amir Sjarifoeddin, qui était membre du PKI. Une autre déficience est le manque d'informations sur les relations de la gauche avec les autres groupes de la société, tels que les groupes catholique et protestant, ou encore le groupe culturel du « Manifeste culturel », en particulier avant 1965, y compris l'attitude politique de la gauche envers ces divers groupes, alors que cela a constitué un paramètre important dans l'émergence, plus tard, d'un sentiment d'antipathie envers la gauche. Il est également surprenant que le livre ne consacre pas la part qu'il mérite au parcours politique de quelques figures dominantes de la gauche, comme par exemple Tan Malaka, qui poursuivit jusqu'à l'étranger la guérilla contre le gouvernement colonial en Indonésie. Vu que le livre est consacré à l'histoire de la gauche indonésienne, qui plus est à l'intention de débutants, une telle lacune laisse le sentiment d'un chaînon manquant.

On peut dire néanmoins que cet ouvrage rend compte méticuleusement $\mathrm{du}$ traumatisme éprouvé par les victimes politiques du gouvernement de Soeharto. Le livre, abondamment illustré, propose au lecteur de mieux concevoir la brutalité et la violence d'État de cette période. Au vu de ce catalogue de scènes de violences entre autres, mutilations ou tortures sexuelles - on peut s'interroger cependant sur la nécessité de montrer dans le détail ces actes de sadisme dans un exposé historique. La question a été posée en relation avec la Shoah, notamment par Hannah Arendt et Claude Lanzmann, à propos de « la banalité du mal » et de « la représentation de l'horreur ». Tous deux considéraient que la représentation minutieuse d'un événement horrible était indésirable, voire indécente, car elle serait de toute façon impuissante à susciter la même terreur au lecteur et qu'elle pourrait, au contraire, conduire à une banalisation de la violence. Dans la figuration d'une scène historique, parfois « less is more ». Vu que l'ouvrage est consacré à l'histoire des mouvements de gauche en Indonésie, il est regrettable qu'il tende à illustrer les souffrances et les tortures plus que les mouvements eux-mêmes. Alors justement qu'un bon nombre de prisonniers politiques et d'exilés ont su préserver leur dignité, des valeurs féministes et de solidarité, et ont même eu le courage de produire des œuvres sous la menace de la tyrannie.

L'histoire de la gauche se limite-t-elle à une perspective dominée par le traumatisme ? Tel est le défi de l'histoire indonésienne face au legs mémoriel de la dictature, qui a dominé la production d'ouvrages historiques durant trois décennies, en construisant un spectre communiste pour les uns et un modèle de victime pour les autres.

Gloria Truly Estrelita

Saskia E. Wieringa and Nursyahbani Katjasungkana, Propaganda and Genocide in Indonesia: Imagined Evil, London - New York: Routledge Contemporary Southeast Asia Series, 2019, xvii-209 p.

Ce n'est qu'en 1978, lorsque le régime de Soeharto commença à libérer les prisonniers de Buru, que Saskia E. Wieringa, étudiante en anthropologie de l'Université d'Amterdam, qui avait vainement cherché jusque là des organisations 